PROCEEDINGS OF THE

AMERICAN MATHEMATICAL SOCIETY

Volume 140, Number 11, November 2012, Pages 3883-3892

S 0002-9939(2012)11243-2

Article electronically published on March 20, 2012

\title{
LINK BETWEEN NOETHERIANITY AND THE WEIERSTRASS DIVISION THEOREM ON SOME QUASIANALYTIC LOCAL RINGS
}

\author{
ABDELHAFED ELKHADIRI
}

(Communicated by Franc Forstneric)

\begin{abstract}
In the setting of well-behaved quasianalytic differentiable systems, we prove that the Weierstrass Division Theorem holds in such system if, and only if, the system is Noetherian.
\end{abstract}

\section{INTRODUCTION}

Let $\mathcal{C}_{k}, k=1,2, \ldots$, be local quasianalytic rings of germs, at the origin in $\mathbb{R}^{k}$, of smooth functions. We suppose that the system $\mathcal{C}=\left\{\mathcal{C}_{k}, k \in \mathbb{N}\right\}$ satisfies some natural properties; see Section 2. We know by [6] that the Weierstrass Division Theorem never holds in such a system if $\mathcal{C}_{k}, k=1,2, \ldots$, is not contained in the ring of germs of real analytic functions. Because of the lack of a Weierstrass Division Theorem, many problems remain open for such rings. For example, we do not know if the $\mathcal{C}_{k}$ are Noetherian rings. The present study may be regarded as an inquiry as to what differences exist between a system $\mathcal{C}=\left\{\mathcal{C}_{k}, k \in \mathbb{N}\right\}$ in which we suppose that a Weierstrass Division Theorem holds and a system in which the $\mathcal{C}_{k}, k=1,2, \ldots$, are Noetherian rings. This question is supported by the following conjecture, asked in the setting of quasianalytic Denjoy-Carlemen classes (see [4, Ch. 7, An algebraic question]).

Conjecture. It may be that a Weierstrass Division Theorem holds in the system $\mathcal{C}=\left\{\mathcal{C}_{k}, k \in \mathbb{N}\right\}$ if and only if the $\mathcal{C}_{k}$ are Noetherian rings.

We remark that in light of the result of [6], where this conjecture is established, we would know that some quasianalytic rings are not Noetherian.

It is clear that if a Weierstrass Division Theorem holds in the system $\mathcal{C}=\left\{\mathcal{C}_{k}, k \in\right.$ $\mathbb{N}\}$, then by repeating standard arguments, we show that the $\mathcal{C}_{k}$ are Noetherian rings; see [12. The aim of this paper is to prove the converse for some Noetherian local rings.

Received by the editors January 4, 2011 and, in revised form, May 9, 2011.

2010 Mathematics Subject Classification. Primary 26E10, 13F25, 32B05, 32B20; Secondary $03 \mathrm{C} 10$

Key words and phrases. Quasianalytic rings, Weierstrass Division Theorem, Noetherian rings.

This work was partially supported by PARS MI33.

(C)2012 American Mathematical Society Reverts to public domain 28 years from publication 


\section{Differentiable System}

Definition 2.1. A differentiable system is a sequence $\mathcal{C}=\left\{\mathcal{C}_{n}, n \in \mathbb{N}\right\}$ such that, for each $n \in \mathbb{N}, \mathcal{C}_{n} \subset \mathcal{E}_{n}$ is a local subring of the ring of germs, at the origin of $\mathbb{R}^{n}$, of $C^{\infty}$ functions. We suppose that, for each $n \in \mathbb{N}, \mathcal{C}_{n}$ is closed under taking derivatives, and the following hold:

$\left(C_{1}\right) \mathbb{R}\left[x_{1}, \ldots, x_{n}\right] \subset \mathcal{C}_{n} \subset \mathcal{E}_{n}$, for each $n \in \mathbb{N}$, where $\mathbb{R}\left[x_{1}, \ldots, x_{n}\right]$ is the ring of polynomials with coefficients in $\mathbb{R}$.

$\left(C_{2}\right)$ The system $\mathcal{C}$ is closed under composition. This means that if $g \in \mathcal{C}_{k}$ and $f=\left(f_{1}, \ldots, f_{k}\right) \in\left(\mathcal{C}_{n}\right)^{k}$ with $f(0)=0$, then $g \circ f \in \mathcal{C}_{n}$.

$\left(C_{3}\right)$ For each $n \in \mathbb{N}, \mathcal{C}_{n}$ is closed under division by coordinates. This means that if $f \in \mathcal{C}_{n}$ and $f=\left(x_{i}-\alpha\right) g$, where $g \in \mathcal{E}_{n}$ and $\alpha \in \mathbb{R}$, then $g \in \mathcal{C}_{n}$.

$\left(C_{4}\right)$ The Implicit Function Theorem for $\mathcal{C}_{n}$ holds in the following sense: Suppose that $f=\left(f_{1}, \ldots, f_{m}\right) \in\left(\mathcal{C}_{n+m}\right)^{m}$ with $f(0,0)=0$. Put $y=\left(y_{1}, \ldots, y_{m}\right)$ and suppose that

$$
\operatorname{det}\left(\frac{\partial f_{i}}{\partial y_{j}}(0,0)\right)_{i, j=1, \ldots, m} \neq 0
$$

Then there is a (unique ) $g=\left(g_{1}, \ldots, g_{m}\right) \in\left(\mathcal{C}_{n}\right)^{m}$ with $g(0)=0$ such that $f(x, g(x))=0$.

Call

$$
\hat{\therefore}: \mathcal{C}_{n} \rightarrow \mathbb{R}\left[\left[x_{1}, \ldots, x_{n}\right]\right]
$$

the map which associates to each $f \in \mathcal{C}_{n}$ its Taylor expansion at the origin. We consider the following conditions:

$\left(C_{5}\right) \hat{\imath}$ is an injective homomorphism.

$\left(C_{6}\right) \mathcal{C}_{n}$ is a Noetherian ring for each $n \in \mathbb{N}$.

Definition 2.2. A differentiable system $\mathcal{C}=\left\{\mathcal{C}_{n}, n \in \mathbb{N}\right\}$ is called quasianalytic [resp. Noetherian] if condition $\left(C_{5}\right)$ holds [resp. if condition $\left(C_{6}\right)$ holds].

Remark 2.3. It is clear that every Noetherian differentiable system is a quasianalytic system.

Example 2.4.

i) If for each $n \in \mathbb{N}, \mathcal{C}_{n}$ is the ring of germs, at the origin in $\mathbb{R}^{n}$, of Nash functions, i.e. algebraic on the ring of polynomials $\mathbb{R}\left[x_{1}, \ldots, x_{n}\right]$, the system $\mathcal{C}=\left\{\mathcal{C}_{n}, n \in \mathbb{N}\right\}$ is a Noetherian differentiable system.

ii) If for each $n \in \mathbb{N}, \mathcal{C}_{n}$ is the ring of germs, at the origin in $\mathbb{R}^{n}$, of real analytic functions, the system $\mathcal{C}=\left\{\mathcal{C}_{n}, n \in \mathbb{N}\right\}$ is a Noetherian differentiable system.

iii) Let $\mathcal{R}$ be a polynomially bounded o-minimal structure which is an expansion of the ordered field of reals. For more details about an o-minimal structure over the field of reals, we refer the reader to [13. We denote by $\mathcal{D}_{n}$ the ring of germs, at the origin in $\mathbb{R}^{n}$, of $C^{\infty}$ definable functions in a neighborhood of the origin in $\mathbb{R}^{n}$. By [8], the system $\mathcal{D}=\left\{\mathcal{D}_{n}, n \in \mathbb{N}\right\}$ is a quasianalytic differentiable system.

iv) Let $M=\left\{M_{p}\right\}_{p=0}^{\infty}$ be an increasing sequence of positive real numbers. We denote by $C_{n}(M) \subset \mathcal{E}_{n}$ the subring of germs of $C^{\infty}$ functions in a neighborhood of the origin which are in the class $M$; see [4] and [10. If we suppose that the class is quasianalytic, then the system $C(M)=\left\{C_{n}(M), n \in \mathbb{N}\right\}$ is a quasianalytic differentiable system. 
In the following, for a differentiable quasianalytic system, we will not distinguish notationally by . between the germ and its image, i.e. its Taylor expansion at the origin.

In particular these conditions on a quasianalytic system imply that the maximal ideal of $\mathcal{C}_{n}$ is $\underline{m}_{n}=\left\{f \in \mathcal{C}_{n} / f(0)=0\right\}=\left(x_{1}, \ldots, x_{n}\right) \mathcal{C}_{n}$ and its completion with the $\underline{m}_{n}$-adic topology is the ring of formal series $\mathbb{R}\left[\left[x_{1}, \ldots, x_{n}\right]\right]$.

\section{Artin APPRoXimation PROPERTy FOR A NOETHERIAN SYSTEM}

Let $\mathcal{C}=\left\{\mathcal{C}_{n}, n \in \mathbb{N}\right\}$ be a differentiable Noetherian system. For each $n \in \mathbb{N}$, the completion of the ring $\mathcal{C}_{n}$ with respect to the $\underline{m}_{n}$-topology is the ring of formal power series $\mathbb{R}\left[\left[x_{1}, \ldots, x_{n}\right]\right]$, which is a regular ring. By [11, Proposition 24], $\mathcal{C}_{n}$ is also a regular ring; hence $\mathcal{C}_{n}$ is an excellent ring [2, Theorem 24]. We see then that the morphism

$$
\mathcal{C}_{n} \rightarrow \mathbb{R}\left[\left[x_{1}, \ldots, x_{n}\right]\right]
$$

is a regular homomorphism [12, Section 1]. The condition $\left(C_{4}\right)$ means that $\mathcal{C}_{n}$ is a Henselian ring. By [12, Theorem 2.4], the Artin approximation property holds for the pair $\left(\mathcal{C}_{n}, \mathbb{R}\left[\left[x_{1}, \ldots, x_{n}\right]\right]\right)$. This means that for every system of polynomial equations $f=0$, where $f=\left(f_{1}, \ldots, f_{q}\right)$ with $f_{i} \in \mathcal{C}_{n}[Y]$ and $Y=\left(Y_{1}, \ldots, Y_{N}\right)$ a set of variables, for each $\nu \in \mathbb{N}$ and each formal solution $\hat{g}=\left(\hat{g}_{1}, \ldots, \hat{g}_{N}\right) \in$ $\left(\mathbb{R}\left[\left[x_{1}, \ldots, x_{n}\right]\right]\right)^{N}$, so that $f(\hat{g})=0$, we can find a solution $g=\left(g_{1}, \ldots, g_{N}\right) \in\left(\mathcal{C}_{n}\right)^{N}$ such that

$$
f(g)=0 \text { and } g-\hat{g} \in \underline{m}_{n}^{\nu} \mathbb{R}\left[\left[x_{1}, \ldots, x_{n}\right]\right]^{N} .
$$

3.1. Monomialization lemma for Noetherian system. We recall a result proved by Eakin-Harris [5, Lemma 5.1] for convergent power series. Here we give its analogue for formal series; the proof is the same.

Lemma 3.1. Let $f \in \mathbb{R}\left[\left[x_{1}, \ldots, x_{n}\right]\right]$. Then there exists $H=\left(x_{1}, M_{2} x_{2}, M_{3} x_{3}, \ldots\right.$, $\left.M_{n} x_{n}\right)$ where for each $j=2, \ldots, n, M_{j}$ is a monomial in only the variables $x_{1}, \ldots, x_{j}$ such that

$$
f\left(x_{1}, M_{2} x_{2}, M_{3} x_{3}, \ldots, M_{n} x_{n}\right)=x_{i_{1}}^{\alpha_{\mu_{1}}} \ldots x_{i_{r}}^{\alpha_{\mu_{r}}} Q
$$

for some unit $Q \in \mathbb{R}\left[\left[x_{1}, \ldots, x_{n}\right]\right], i_{1}, \ldots i_{r} \in\{1, \ldots, n\}$ and $\alpha_{\mu_{1}}, \ldots, \alpha_{\mu_{r}} \in \mathbb{N}$.

Proposition 3.2. Let $\mathcal{C}=\left\{\mathcal{C}_{n}, n \in \mathbb{N}\right\}$ be a differentiable Noetherian system, and let $\varphi \in \mathcal{C}_{n}$. Then there exits $H=\left(x_{1}, M_{2} x_{2}, M_{3} x_{3}, \ldots, M_{n} x_{n}\right)$ where for each $j=2, \ldots, n, M_{j}$ is a monomial in only the variables $x_{1}, \ldots, x_{j}$ such that

$$
\varphi\left(x_{1}, M_{2} x_{2}, M_{3} x_{3}, \ldots, M_{n} x_{n}\right)=x_{i_{1}}^{\alpha_{\mu_{1}}} \ldots x_{i_{r}}^{\alpha_{\mu_{r}}} Q
$$

for some unit $Q \in \mathcal{C}_{n}, i_{1}, \ldots i_{r} \in\{1, \ldots, n\}$ and $\alpha_{\mu_{1}}, \ldots, \alpha_{\mu_{r}} \in \mathbb{N}$.

Proof. If $\varphi \in \mathcal{C}_{n}$, by Lemma 3.1 , there exists $H=\left(x_{1}, M_{2} x_{2}, M_{3} x_{3}, \ldots, M_{n} x_{n}\right)$ where for each $j=2, \ldots, n, M_{j}$ is a monomial in only the variables $x_{1}, \ldots, x_{j}$ such that

$$
\hat{\varphi}\left(x_{1}, M_{2} x_{2}, M_{3} x_{3}, \ldots, M_{n} x_{n}\right)=x_{i_{1}}^{\alpha_{\mu_{1}}} \ldots x_{i_{r}}^{\alpha_{\mu_{r}}} \hat{Q}
$$

for some unit $\hat{Q} \in \mathbb{R}\left[\left[x_{1}, \ldots, x_{n}\right]\right], i_{1}, \ldots i_{r} \in\{1, \ldots, n\}$ and $\alpha_{\mu_{1}}, \ldots, \alpha_{\mu_{r}} \in \mathbb{N}$. We consider the equation $E(Z)$ :

$$
x_{i_{1}}^{\alpha_{\mu_{1}}} \ldots x_{i_{r}}^{\alpha_{\mu_{r}}} Z-\varphi\left(x_{1}, M_{2} x_{2}, M_{3} x_{3}, \ldots, M_{n} x_{n}\right)=0 .
$$


We see that $E(Z) \in \mathcal{C}_{n}[Z]$ and $\hat{Q}$ is a formal solution of this equation. By the Artin Approximation Theorem, if $\nu \in \mathbb{N}^{*}$, there exists $Q \in \mathcal{C}_{n}$ that is a solution of the equation $E(Z)$ such that $Q-\hat{Q} \in \underline{m}_{n}^{\nu} \mathbb{R}\left[\left[x_{1}, \ldots, x_{n}\right]\right]$, hence the result since $\nu \geq 1$.

\section{EAKIN-HARRIS PROPERTY}

In this section, we fix a quasianalytic differentiable system $\mathcal{C}=\left\{\mathcal{C}_{n}, n \in \mathbb{N}\right\}$. We put $x=\left(x_{1}, \ldots, x_{n}\right)$ and $y=\left(y_{1}, \ldots, y_{k}\right)$. We are concerned here with local homomorphisms $\Phi: \mathcal{C}_{n} \rightarrow \mathcal{C}_{k}$, i.e. homomorphisms such that $\Phi\left(\underline{m}_{n}\right) \subset \underline{m}_{k}$. The generic rank of $\Phi$, denoted $r k(\Phi)$, is the rank of the Jacobian matrix $\left[\frac{\partial \Phi\left(x_{i}\right)}{\partial y_{j}}\right]$, considered as a matrix over the quotient field of $\mathcal{C}_{k}$. Recall that $\mathcal{C}_{k}$ is a domain by condition $\left(C_{5}\right)$. Since $\Phi$ is a local homomorphism, we consider its natural extension to the completion $\hat{\Phi}: \mathbb{R}\left[\left[x_{1}, \ldots, x_{n}\right]\right] \rightarrow \mathbb{R}\left[\left[y_{1}, \ldots, y_{k}\right]\right]$. It is well known that if $r k(\Phi)=n$, then $\Phi$ is injective. In [9], we can find an example (Osgood's example) of an injective homomorphism $\Phi$ for which $r k(\Phi)<n$. Thus the condition is not necessary.

We let

$$
\Phi_{*}: \frac{\mathbb{R}\left[\left[x_{1}, \ldots, x_{n}\right]\right]}{\mathcal{C}_{n}} \rightarrow \frac{\mathbb{R}\left[\left[y_{1}, \ldots, y_{k}\right]\right]}{\mathcal{C}_{k}}
$$

be the homomorphism of groups induced by $\Phi$ and $\hat{\Phi}$ in the obvious manner.

Definition 4.1. We say that the homomorphism $\Phi$ is strongly injective if the homomorphism $\Phi_{*}$ is injective.

In the analytic setting, i.e. when each $\mathcal{C}_{n}$ is the ring of germs of real analytic functions, Eakin and Harris [5] showed that $\Phi$ is strongly injective if and only if $r k(\Phi)=n$. Their result extends a result of Abhyankar and van der Put [1].

This result justifies the following definition:

Definition 4.2. We say that a local morphism $\Phi: \mathcal{C}_{n} \rightarrow \mathcal{C}_{k}$ has the Eakin-Harris property if $r k(\Phi)=n$ implies $\Phi$ is strongly injective.

It is shown in [7] that if every morphism $\Phi: \mathcal{C}_{n} \rightarrow \mathcal{C}_{k}$ has the Eakin-Harris property, then the system $\mathcal{C}=\left\{\mathcal{C}_{n}\right\}$ is contained in the analytic system.

Lemma 4.3. Let $\Phi: \mathcal{C}_{n} \rightarrow \mathcal{C}_{k}$ and $\Psi: \mathcal{C}_{k} \rightarrow \mathcal{C}_{l}$ be local homomorphisms. If $\Psi \circ \Phi$ is strongly injective, then $\Phi$ is strongly injective.

Proof. Follows from the definition.

Remark 4.4. Isomorphisms are strongly injective.

Recall that a local homomorphism $u: A \rightarrow B$ between local rings is called finite if $B$ is a finite module over the ring $u(A)$.

Lemma 4.5 ([1]). Let $\mathcal{C}=\left\{\mathcal{C}_{n}, n \in \mathbb{N}\right\}$ be a differentiable Noetherian system. If $\Phi: \mathcal{C}_{n} \rightarrow \mathcal{C}_{k}$ is injective and finite, then $\hat{\Phi}$ is injective and finite and $\Phi$ is strongly injective.

Proof. Since each $\mathcal{C}_{k}$ is a Zariski ring, the lemma follows from [13, Chapter VIII, Theorem 9].

In the following we identify local homomorphisms $\Phi: \mathcal{C}_{n} \rightarrow \mathcal{C}_{k}$ defined by $\Phi(f)=$ $f\left(\varphi_{1}, \ldots, \varphi_{n}\right), \varphi_{i} \in \mathcal{C}_{k}, i=1, \ldots, n$, by $\left(\varphi_{1}, \ldots, \varphi_{n}\right)$ and we write $\Phi=\left(\varphi_{1}, \ldots, \varphi_{n}\right)$. 
We consider the local homomorphism

$$
e=\left(y_{1} y_{2}, y_{2}, \ldots, y_{k}\right): \mathcal{C}_{k} \rightarrow \mathcal{C}_{k} .
$$

For $d \in \mathbb{N}^{*}$ we also consider the local homomorphism

$$
r_{d}=\left(y_{1}^{d}, y_{2}, \ldots, y_{k}\right): \mathcal{C}_{k} \rightarrow \mathcal{C}_{k}
$$

It is clear that homomorphisms $e, r_{d}(d \in \mathbb{N})$ are injective and $r k\left(r_{d}\right)=r k(e)=$ $k$.

Remark 4.6. Let $P=\sum_{\omega} a_{\omega} y_{1}^{\omega_{1}} y_{2}^{\omega_{2}} \ldots y_{k}^{\omega_{k}}$ be a polynomial. There exists $N \in \mathbb{N}$ such that, for each $\omega=\left(\omega_{1}, \ldots, \omega_{k}\right) \in \mathbb{N}^{k}$ with $a_{\omega} \neq 0, N+\omega_{2} \geq \omega_{1}$. We have then $y_{2}^{N} P=e(Q)$, where $Q=\sum_{\omega} a_{\omega} y_{1}^{\omega_{1}} y_{2}^{N+\omega_{2}-\omega_{1}} y_{3}^{\omega_{3}} \ldots y_{k}^{\omega_{k}}$.

Definition 4.7. We say that the quasianalytic differentiable system $\mathcal{C}=\left\{\mathcal{C}_{n}, n \in\right.$ $\mathbb{N}\}$ is well behaved if the homomorphisms $e$ and $r_{d}$ are strongly injective.

Proposition 4.8. The systems considered in i) and ii) of Example 2.4 are well behaved.

Proof.

(1) Analytic system.

Let $\hat{f}=\sum_{\alpha \in \mathbb{N}^{n}} a_{\alpha} x_{1}^{\alpha_{1}} \ldots x_{n}^{\alpha_{n}}, \alpha=\left(\alpha_{1}, \ldots, \alpha_{n}\right)$, be a formal power series such that $e(\hat{f})$ is a convergent series. Hence there exist $r, M \in \mathbb{R}_{+}^{*}, r<1$, such that for every $\alpha \in \mathbb{N}^{n},\left|a_{\alpha}\right| r^{|\alpha|+\alpha_{1}} \leq M$, where $|\alpha|=\alpha_{1}+\ldots+\alpha_{n}$. We have then, for every $\alpha \in \mathbb{N}^{n},\left|a_{\alpha}\right| r^{2|\alpha|} \leq M$, which proves that $\hat{f}$ is a convergent power series; hence the homomorphism $e$ is strongly injective. It is also an elementary calculation to show that the homomorphism $r_{d}, d \in \mathbb{N}$, is strongly injective. Hence the analytic system is well behaved.

(2) Nash system.

Let $\hat{f}=\sum_{\alpha \in \mathbb{N}^{n}} a_{\alpha} x_{1}^{\alpha_{1}} \ldots x_{n}^{\alpha_{n}}$ be a formal power series such that $e(\hat{f})$ is algebraic on the ring of polynomials $\mathbb{R}\left[x_{1}, \ldots, x_{n}\right]$. It is known already that $e(\hat{f})$ is a convergent series (the ring of convergent power series is algebraically closed in the ring of formal power series); hence $\hat{f}$ is also a convergent power series. We have:

$$
P_{q}(e(\hat{f}))^{q}+\ldots+P_{1} e(\hat{f})+P_{0}=0,
$$

with all the polynomials $P_{j} \neq 0$. By Remark 4.6, there exist $m \in \mathbb{N}$ and polynomials $Q_{j}, j=1, \ldots, q$, such that $x_{2}^{m} P_{j}=e\left(Q_{j}\right)$. By multiplying the equation $(*)$ by $x_{2}^{m}$ and since $e$ is injective, we have $Q_{q} \hat{f}^{q}+\ldots+Q_{0}=0$; hence $\hat{f}$ is algebraic on the ring of polynomials, which proves that the morphism $e$ is strongly injective.

Now, let $\hat{f}=\sum_{\alpha \in \mathbb{N}^{n}} a_{\alpha} x_{1}^{\alpha_{1}} \ldots x_{n}^{\alpha_{n}}$ be a formal power series such that $r_{d}(\hat{f})$ is algebraic on the ring of polynomials. Since the Weierstrass Division Theorem is true in the ring of Nash functions (see [3, 8.2.8]), we divide $r_{d}(\hat{f})$ by the polynomial $x_{1}^{d}-T$, where $T$ is an auxiliary variable,

$$
r_{d}(\hat{f})=\left(x_{1}^{d}-T\right) Q(x, T)+\sum_{j=1}^{d} b_{j}\left(x_{2}, \ldots, x_{n}, T\right) x_{1}^{d-j},
$$

where $Q(x, T), b_{j}\left(x_{2}, \ldots, x_{n}, T\right)$ are algebraic on the ring of polynomials, $j=1, \ldots, d$. 
We also divide the formal series $\hat{f}$ by the polynomial $x_{1}-T$ in the ring of formal series $\mathbb{R}\left[\left[x_{1}, \ldots, x_{n}, T\right]\right]$,

$$
\hat{f}=\left(x_{1}-T\right) Q_{1}(x, T)+W\left(x_{2}, x_{3}, \ldots, x_{n}, T\right),
$$

where $Q_{1}(x, T) \in \mathbb{R}\left[\left[x_{1}, \ldots, x_{n}, T\right]\right], W\left(x_{2}, x_{3}, \ldots, T\right) \in \mathbb{R}\left[\left[x_{2}, \ldots, x_{n}, T\right]\right]$.

We have then

$\hat{f}\left(x_{1}^{d}, x_{2}, \ldots, x_{n}\right)=\left(x_{1}^{d}-T\right) Q_{1}\left(x_{1}^{d}, x_{2}, \ldots, x_{n}, T\right)+W\left(x_{2}, x_{3}, \ldots, x_{n}, T\right)$.

Since the division is unique in $\mathbb{R}\left[\left[x_{1}, \ldots, x_{n}, T\right]\right]$, we see that

$$
Q(x, T)=Q_{1}\left(x_{1}^{d}, x_{2}, \ldots, x_{n}, T\right)
$$

and

$$
\sum_{j=1}^{d} b_{j}\left(x_{2}, \ldots, x_{n}, T\right) x_{1}^{d-j}=W\left(x_{2}, x_{3}, \ldots, x_{n}, T\right) .
$$

Hence $b_{1}=\ldots=b_{d-1}=0$, and $W\left(x_{2}, x_{3}, \ldots, x_{n}, T\right)=b_{d}\left(x_{2}, \ldots, x_{n}, T\right)$, but, from $(* *)$, we have $W\left(x_{2}, x_{3}, \ldots, x_{n}, x_{1}\right)=\hat{f}\left(x_{1}, \ldots, x_{n}\right)$, hence the result.

Remark 4.9. For each $l \in\{1,2, \ldots, k-1\}$, the local homomorphism $H=$ $\left(y_{1}, y_{2}, \ldots, y_{l}, y_{l} y_{l+1}, \ldots, y_{l} y_{k}\right): \mathcal{C}_{k} \rightarrow \mathcal{C}_{k}$ is a finite compositions of $e$ and permutations.

\section{EAKIN-HARRIS PROPERTY FOR DIfFERENTIABLE NOETHERIAN SYSTEM}

We prove in this section a version of a theorem proved by Eakin and Harris [5]. This result is about local homomorphisms of rings in a given well-behaved differentiable Noetherian system. The proof is inspired by the proof of a similar result in the setting of an analytic system, proved by Eakin and Harris [5].

We put $x=\left(x_{1}, \ldots, x_{n}\right)$ and $y=\left(y_{1}, \ldots, y_{k}\right)$. If $\mathcal{C}=\left\{\mathcal{C}_{n}, n \in \mathbb{N}\right\}$ is a quasianalytic differentiable system, let $\Phi: \mathcal{C}_{n} \rightarrow \mathcal{C}_{k}$ be a local homomorphism.

Definition 5.1. An admissible modification of $\Phi$ is a homomorphism $\tilde{\Phi}$ related to $\Phi$ in one of the following ways:

(i) There is an isomorphism $\Gamma: \mathcal{C}_{n} \rightarrow \mathcal{C}_{n}$ such that $\Phi \circ \Gamma=\tilde{\Phi}$.

(ii) There is a homomorphism $\Psi: \mathcal{C}_{k} \rightarrow \mathcal{C}_{k}$ with $r k(\Psi)=k$ such that $\Psi \circ \Phi=\tilde{\Phi}$.

(iii) There is a strongly injective homomorphism $\omega: \mathcal{C}_{n} \rightarrow \mathcal{C}_{n}$ with $r k(\omega)=n$ such that $\Phi=\tilde{\Phi} \circ \omega$.

Remark 5.2. Let $\tilde{\Phi}$ be an admissible modification of $\Phi$. Then

(i) $r k(\tilde{\Phi})=r k(\Phi)$.

(ii) $\tilde{\Phi}$ strongly injective $\Rightarrow \Phi$ strongly injective.

From now on, we suppose that the system $\mathcal{C}=\left\{\mathcal{C}_{n}, n \in \mathbb{N}\right\}$ is a well-behaved Noetherian differentiable system. Let $\Phi: \mathcal{C}_{n} \rightarrow \mathcal{C}_{k}, \Phi=\left(\varphi_{1}, \ldots, \varphi_{n}\right)$, be a local homomorphism. We suppose $\varphi_{1} \neq 0$.

Lemma 5.3. There exist $d \in \mathbb{N}^{*}$ and a homomorphism $H: \mathcal{C}_{k} \rightarrow \mathcal{C}_{k}$ such that

$$
H\left(\varphi_{1}\right)=c_{1} y_{1}^{d}+c_{2} y_{2}^{d}+\ldots+c_{k} y_{k}^{d}+\psi
$$

where $c_{1}, c_{2}, \ldots, c_{k} \in \mathbb{R}, c_{1} \neq 0$ and $\psi \in \mathcal{C}_{k}$ such that $\hat{\psi}=\{$ mixed terms of degree $d\}$ $+\sum_{|\omega|>d} a_{\omega} y_{1}^{\omega_{1}} \ldots y_{k}^{\omega_{k}}$. 
Proof. $\hat{\varphi}_{1}=\sum_{\nu=0}^{\infty} P_{\nu}\left(y_{1}, \ldots, y_{k}\right)$, where $P_{\nu}\left(y_{1}, \ldots, y_{k}\right)$ is a homogeneous polynomial of degree $\nu, \nu=0,1, \ldots$ Let $d \in \mathbb{N}$ be the least integer such that $P_{d} \neq 0$. We have then

$$
\hat{\varphi}_{1}=P_{d}\left(y_{1}, \ldots, y_{k}\right)+\sum_{\nu>d}^{\infty} P_{\nu}\left(y_{1}, \ldots, y_{k}\right) .
$$

Let $\left(\lambda_{i j=1}\right)_{i, j=1}^{k}$ be a nonsingular matrix of scalars such that $P_{d}\left(\lambda_{11}, \ldots, \lambda_{k 1}\right) \neq$ 0 . We define an isomorphism $H: \mathcal{C}_{k} \rightarrow \mathcal{C}_{k}$ by

$$
H=\left(\sum_{l=1}^{k} \lambda_{1 l} y_{l}, \sum_{l=2}^{k} \lambda_{2 l} y_{l}, \ldots, \sum_{l=1}^{k} \lambda_{k l} y_{l}\right) .
$$

We put $\tilde{\varphi_{1}}:=H\left(\varphi_{1}\right) \in \mathcal{C}_{k}$. We see then that

$$
\hat{\hat{\varphi}_{1}}=c_{1} y_{1}^{d}+c_{2} y_{2}^{d}+\ldots+c_{k} y_{k}^{d}+\{\text { mixed terms of degree } d\}+\sum_{|\omega|>d} b_{\omega} y_{1}^{\omega_{1}} \ldots y_{k}^{\omega_{k}},
$$

where $c_{1}=P_{d}\left(\lambda_{11}, \ldots, \lambda_{k 1}\right), c_{2}=P_{d}\left(\lambda_{12}, \ldots, \lambda_{k 2}\right), \ldots, c_{k}=P_{d}\left(\lambda_{1 k}, \ldots, \lambda_{k k}\right)$.

If we put $\hat{\gamma}:=\sum_{|\omega|>d} b_{\omega} y_{1}^{\omega_{1}} \ldots y_{k}^{\omega_{k}}$, we see that $\hat{\gamma}$ is a formal solution of the equation

$$
Z+c_{1} y_{1}^{d}+c_{2} y_{2}^{d}+\ldots+c_{k} y_{k}^{d}+\{\text { mixed terms of degree } d\}-\tilde{\varphi_{1}}=0
$$

By the Artin Approximation Theorem, if $\nu \in \mathbb{N}, \nu>d$, there exists $\psi \in \mathcal{C}_{k}$ that is a solution of the equation (2) such that $\hat{\psi}-\hat{\gamma} \in \underline{m}_{n}^{\nu} \mathbb{R}\left[\left[x_{1}, \ldots, x_{n}\right]\right]$, which proves the lemma.

We consider $H_{1}=\left(y_{1}, y_{1} y_{2}, \ldots, y_{1} y_{k}\right): \mathcal{C}_{k} \rightarrow \mathcal{C}_{k}$. We have

$$
H_{1} H\left(\varphi_{1}\right)\left(y_{1}, \ldots, y_{k}\right)=y_{1}^{d} \psi_{1},
$$

for some unit $\psi_{1} \in \mathcal{C}_{k}$.

Since $\psi_{1}$ is a unit, there is $\psi_{2} \in \mathcal{C}_{k}$ such that $\psi_{2}^{d} \psi_{1}=1$. Now we define $H_{2}:=$ $\left(y_{1} \psi_{2}, y_{2}, \ldots, y_{k}\right): \mathcal{C}_{k} \rightarrow \mathcal{C}_{k}$. We have $H_{2} H_{1} H\left(\varphi_{1}\right)\left(y_{1}, \ldots, y_{k}\right)=y_{1}^{d}$.

Remark 5.4. We remark that the homomorphism

$$
\left(y_{1}^{d}, H_{2} H_{1} H\left(\varphi_{1}\right), \ldots, H_{2} H_{1} H\left(\varphi_{n}\right)\right)
$$

is an admissible modification of $\Phi$. Since our system is well behaved, we notice, also, that the homomorphism $\left(y_{1}, H_{2} H_{1} H\left(\varphi_{1}\right), \ldots, H_{2} H_{1} H\left(\varphi_{n}\right)\right)$ is an admissible modification of $\left(y_{1}^{d}, H_{2} H_{1} H\left(\varphi_{1}\right), \ldots, H_{2} H_{1} H\left(\varphi_{n}\right)\right)$.

Theorem 5.5. If $r k(\Phi)=s$, then there is a finite sequence $\Phi_{1}, \ldots, \Phi_{l}$ of homomorphisms $\Phi_{i}: \mathcal{C}_{n} \rightarrow \mathcal{C}_{k}$ such that $\Phi_{1}=\Phi, \Phi_{i+1}$ is related to $\Phi_{i}$ by an admissible modification, $i=1, \ldots, l-1$, and $\Phi_{l}=\left(y_{1}, \ldots, y_{s}, 0,0, \ldots 0\right)$.

Proof. By the above remark, we can suppose that $\Phi=\left(y_{1}, \varphi_{2}, \ldots \varphi_{n}\right)$. Assume we have found a sequence $\left(\Phi_{i}\right)_{i=1}^{N}$ of admissible modifications such that $\Phi_{N}=$ $\left(y_{1}, y_{2}, \ldots, y_{j}, \varphi_{j+1}, \ldots, \varphi_{n}\right)$ for some integer $j$. Notice $j \leq s$, since $r k\left(\Phi_{N}\right)=s$. We consider two cases:

Case $j<s$. We may assume that one of the germs, $\varphi_{j+1}, \ldots, \varphi_{k}$, depends on at least one of the remaining variables $y_{j+1}, \ldots, y_{k}$. If not, we consider the homomorphism $\Gamma=\left(x_{1}, \ldots, x_{j}, x_{j+1}-\varphi_{j+1}, x_{j+2}, \ldots, x_{n}\right)$. We see then that $\Phi_{N} \circ$ $\Gamma=\left(y_{1}, \ldots, y_{j}, 0, \varphi_{j+2}, \ldots, \varphi_{n}\right)$ is an admissible modification of $\Phi_{N}$. We continue 
with $\varphi_{j+2}$. At the end we find an admissible modification of $\Phi_{N}$ of the form $\left(y_{1}, \ldots, y_{j}, 0 \ldots, 0\right)$, which is a contradiction since $r k\left(\Phi_{N}\right)=s$.

We can then suppose that $\varphi_{j+1}$ depends on at least one of the variables $y_{j+1}, \ldots, y_{k}$. We have

$$
\hat{\varphi}_{j+1}=P_{d}\left(y_{j+1}, \ldots, y_{k}\right)+\hat{\psi}
$$

where $P_{d} \in \mathcal{C}_{j}\left[y_{j+1}, \ldots, y_{k}\right]$ is a homogenous polynomial of degree $d, \hat{\psi} \in$ $\left(y_{j+1}, \ldots, y_{k}\right)^{d+1} \mathbb{R}\left[\left[y_{1}, \ldots, y_{k}\right]\right]$. We have then $\hat{\psi}=\sum_{l=1}^{q} \lambda_{l} \hat{\beta}_{l}$, where $\lambda_{l} \in$ $\left(y_{j+1}, \ldots, y_{k}\right)^{d+1} \in \mathbb{R}\left[y_{1}, \ldots, y_{k}\right]$ and $\hat{\beta}_{l} \in \mathbb{R}\left[\left[y_{1}, \ldots, y_{k}\right]\right]$. We put

$$
E\left(Z, Z_{1}, \ldots, Z_{q}\right)=\left(\varphi_{j+1}-P_{d}\left(y_{j+1}, \ldots, y_{k}\right)-Z, Z-\sum_{l=1}^{q} \lambda_{l} Z_{l}\right),
$$

$E\left(Z, Z_{1}, \ldots, Z_{q}\right) \in\left(\mathcal{C}_{k}\left[Z, Z_{1}, \ldots, Z_{q}\right]\right)^{2}$.

We see that $\left(\hat{\psi}, \hat{\beta}_{1}, \ldots, \hat{\beta}_{q}\right)$ is a formal solution of the equation

$$
E\left(Z, Z_{1}, \ldots, Z_{q}\right)=0 .
$$

By the Artin Approximation Theorem, there exists $\left(\psi, \beta_{1}, \ldots, \beta_{q}\right) \in\left(\mathcal{C}_{k}\right)^{q+1}$ that is a solution of the equation $E\left(Z, Z_{1}, \ldots, Z_{q}\right)=0$. We have then that

$$
\varphi_{j+1}=P_{d}\left(y_{j+1}, \ldots, y_{k}\right)+\psi
$$

with $\psi \in\left(y_{j+1}, \ldots, y_{k}\right)^{d+1} \mathcal{C}_{k}$.

Let $\left(\lambda_{i, \nu}\right)_{\nu=j+1}^{k}$ be a nonsingular matrix of scalars and define the homomorphism $H: \mathcal{C}_{k} \rightarrow \mathcal{C}_{k}$ by

$$
H=\left(y_{1}, \ldots, y_{j}, \sum_{\nu=j+1}^{k} \lambda_{i, \nu} y_{\nu}, \ldots, \sum_{\nu=j+1}^{k} \lambda_{k, \nu} y_{\nu}\right) .
$$

We see then that

$$
H\left(\varphi_{j+1}\right)=c_{j+1} y_{j+1}^{d}+\ldots+c_{k} y_{k}^{d}+\{\text { mixed terms of degree } d\}+H(\psi),
$$

where

We put

$$
c_{l}=P_{d}\left(\lambda_{l, j+1}, \lambda_{l, j+2}, \ldots, \lambda_{l, k}\right), l=j+1, \ldots, k
$$

$$
Q=c_{j+2} y_{j+2}^{d}+\ldots+c_{k} y_{k}^{d}+\{\text { mixed terms of degree } d\} .
$$

Let us note that each term of $Q$ is divisible by at least one of $y_{j+2}, \ldots, y_{k}$. $\neq 0$.

We may choose the matrix $\left(\lambda_{i, \nu}\right)_{\nu=j+1}^{k}$ so that $P_{d}\left(\lambda_{j+1, j+1}, \lambda_{j+1, j+2}, \ldots, \lambda_{j+1, k}\right)$

Define $G: \mathcal{C}_{k} \rightarrow \mathcal{C}_{k}$ by

$$
G=\left(y_{1}, \ldots, y_{j+1}, y_{j+1} y_{j+2}, \ldots, y_{j+1} y_{k}\right) .
$$

We see that $y_{j+1}^{d}$ divides $G \circ H\left(\varphi_{j+1}\right)$. Since $G \circ H\left(\Phi_{N}\right)$ is an admissible modification of $\Phi_{N}$, we can suppose that $y_{j+1}^{d}$ divides $\varphi_{j+1}$ and $\varphi_{j+1}$ is still of the form (3).

On the other hand, by Proposition 3.2, there exists $H_{1}: \mathcal{C}_{k} \rightarrow \mathcal{C}_{k}$,

$$
H_{1}=\left(y_{1}, y_{1}^{e_{1,2}} y_{2}, y_{1}^{e_{1,3}} y_{2}^{e_{2,3}} y_{3}, \ldots, y_{1}^{e_{1, k}} y_{2}^{e_{2, k}} y_{3}^{e_{3, k}} \ldots y_{k-1}^{e_{k-1, k}} y_{k}\right),
$$

such that $H_{1}\left(\varphi_{j+1}\right)=($ monomial $) \cdot Q_{1}$ for some unit $Q_{1} \in \mathcal{C}_{k}$.

From (3), we have

$$
H_{1}\left(\varphi_{j+1}\right)=H_{1}\left(c_{j+1}\right)\left(y_{1}^{e_{1, j+1}} y_{2}^{e_{2, j+1}} \ldots y_{j}^{e_{j, j+1}} y_{j+1}\right)^{d}+H_{1}\left(Q_{1}\right)+H_{1}(\psi) .
$$


$H_{1}\left(c_{j+1}\right) \in \mathcal{C}_{j}, H_{1}\left(c_{j+1}\right) \neq 0$, and each term of $H_{1}\left(Q_{1}\right)$ is divisible by at least one of $y_{j+2}, \ldots, y_{k}$ and $H_{1}(\psi) \in\left(y_{j+1}, \ldots, y_{k}\right)^{d+1} \mathcal{C}_{k}$.

By (4), we see then that none of $y_{j+2}, \ldots, y_{k}$ divides $H_{1}\left(\varphi_{j+1}\right)$. On the other hand, we know that $y_{j+1}^{d}$ divides $\varphi_{j+1}$, and hence $y_{j+1}^{d}$ divides $H_{1}\left(\varphi_{j+1}\right)$. But we have $H_{1}\left(\varphi_{j+1}\right)=($ monomial $) \cdot Q_{1}$; hence

$$
H_{1}\left(\varphi_{j+1}\right)=\left(y_{1}^{\epsilon_{1, j+1}} y_{2}^{\epsilon_{2, j+1}} \ldots y_{j}^{\epsilon_{j, j+1}} y_{j+1}^{\epsilon_{j+1, j+1}}\right) Q_{1} \text {. }
$$

Thus we have modified $\Phi_{N}$ to an $H_{1}\left(\Phi_{N}\right)$ of the form

$H_{1}\left(\Phi_{N}\right)=\left(y_{1}, y_{1}^{e_{1,2}} y_{2}, \ldots, y_{1}^{e_{1, j}} y_{2}^{e_{2, j}} \ldots y_{j-1}^{e_{j-1, j}} y_{j}, y_{1}^{\epsilon_{1, j+1}} \ldots y_{j+1}^{\epsilon_{j+1, j+1}} Q_{1}, \varphi_{j+2}, \ldots, \varphi_{n}\right)$.

Now we may absorb the unit $Q_{1}$ into $y_{j+1}$ as above. By successive applications of morphisms $e$ and $r_{d}$ we have modified $\Phi_{N}$ to the form

$$
\Phi_{N^{\prime}}=\left(y_{1}, \ldots, y_{j}, y_{j+1}, \varphi_{j+2}, \varphi_{n}\right) .
$$

Case $j=s$. If $\Phi_{N}=\left(y_{1}, \ldots, y_{s}, \varphi_{s+1}, \ldots, \varphi_{n}\right)$, then for each $l=s+1, \ldots, n$, $\varphi_{l}$ is independent of all $y_{s+1}, \ldots, y_{k}$. Otherwise we could modify $\Phi_{N}$ by the above procedure to $\Phi_{N}=\left(y_{1}, \ldots, y_{s}, y_{s+1}, \varphi_{s+2}, \ldots, \varphi_{n}\right)$, but $r k\left(\Phi_{N}\right)=s+1>s$.

Define $\Gamma: \mathcal{C}_{n} \rightarrow \mathcal{C}_{n}$ by

$$
\Gamma=\left(x_{1}, \ldots, x_{s}, x_{s+1}-\varphi_{s+1}\left(x_{1}, \ldots, x_{s}\right), \ldots, x_{n}-\varphi_{n}\left(x_{1}, \ldots, x_{s}\right)\right) .
$$

We see then that $\Phi_{n} \circ \Gamma$ is defined by $\left(y_{1}, \ldots, y_{s}, 0, \ldots, 0\right)$, and hence the theorem is proved.

Corollary 5.6. Let $\Phi: \mathcal{C}_{n} \rightarrow \mathcal{C}_{k}$ be a local homomorphism. Suppose that $r k(\Phi)=$ $n$. Then $\Phi$ is strongly injective.

Proof. By the above theorem we can modify $\Phi$ by successive admissible modifications to the form $\left(y_{1}, y_{2}, \ldots, y_{n}\right)$, which is trivially strongly injective.

Corollary 5.7. The Weierstrass Division Theorem holds in the well-behaved Noetherian differentiable system $\mathcal{C}=\left\{\mathcal{C}_{n}, n \in \mathbb{N}\right\}$.

Proof. First, every Noetherian differentiable system is a quasianalytic differentiable system. By Corollary 5.6, every local homomorphism $\Phi: \mathcal{C}_{n} \rightarrow \mathcal{C}_{k}$ with $r k(\Phi)=n$ is strongly injective, hence the result by Theorem 4.1 of $[7$.

Corollary 5.8. Every well-behaved Noetherian differentiable system is continued in the analytic system.

Proof. By Corollary 5.7, the Weierstrass Division Theorem holds in this system. We deduce the result by [6].

\section{REFERENCES}

[1] S.S. Abhyankar and M. van der Put. Homomorphisms of analytic local rings. J. Reine Angew. Math. 242 (1970), 26-60. MR0260729 (41:5353)

[2] C. Andradas, L. Bröcker and J.M. Ruiz. Constructible sets in real geometry. Ergeb. Math., Volume 33, Springer (1996). MR1393194 (98e:14056)

[3] J. Bochnak, M. Coste, and M.-F. Roy. Géométrie algébrique réelle. Springer-Verlag, Berlin and Heidelberg (1987). MR949442 (90b:14030)

[4] C.L. Childress, Weierstrass division in quasianalytic local rings. Can. J. Math., Vol. XXVIII, No. 5, 1976, pp. 938-953. MR0417441(54:5491)

[5] P.M. Eakin and G.A. Harris, When $F(f)$ convergent implies $f$ is convergent, Math. Ann. 229, 201-210 (1977). MR0444651 (56:3001) 
[6] A. Elkhadiri and H. Sfouli, Weierstrass division theorem in quasianalytic local rings, Studia Mathematica 185 (1) (2008). MR2380000

[7] A. Elkhadiri, Homomorphism of quasianalytic local rings, Proc. Am. Math. Soc. 138, No. 4, 1433-1438 (2010). MR2578536 (2011a:32008)

[8] C. Miller, Infinite differentiability in polynomially bounded o-minimal structures. Proc. Amer. Math. Soc. 123 (1995), 2551-2555. MR1257118 (95j:03069)

[9] W.F. Osgood, On functions of several complex variables. Trans. Amer. Math. Soc. 17 (1916), 1-8. MR.1501027

[10] W. Rudin, Real and complex analysis. McGraw-Hill, New York (1966). MR0210528 (35:1420)

[11] J.-P. Serre, Algèbre locale. Multiplicités, Lecture Notes in Mathematics, 11, Springer-Verlag, Berlin-New York (1965). MR0201468 (34:1352)

[12] B. Teissier, Résultat récents sur l'approximation des morphismes en algèbre commutative. Séminaire Bourbaki, 46ème année, 1993-94, Astérisque 227 (1995), no. 784. MR 1321650 (96c:13023)

[13] J.-Cl. Tougeron, Idéaux des fonctions différentiables. Ergebnisse der Mathematik und ihrer Grenzgebiete, Band 71, Springer-Verlag (1972). MR0440598 (55:13472)

[14] L. Van Den Dries, Tame topology and o-minimal structures. London Mathematical Society, Lecture Note Series, 248. Cambridge University Press, Cambridge (1998). MR 1633348 (99j:03001)

[15] O. Zariski and P. Samuel, Commutative Algebra, Volume II, D. Van Nostrand (1960). MR0120249(22:11006)

Department of Mathematics, Faculty of Sciences, University Ibn Tofail, B.P. 133, KÉNITRA, Morocco

E-mail address: kabdelhafed@hotmail.com 